Sequence-definition from controlled polymerization: the next generation of materials

Peer-reviewed author version

DE NEVE, Jeroen; HAVEN, Joris; MAES, Lowie \& JUNKERS, Tanja (2018)

Sequence-definition from controlled polymerization: the next generation of materials.

In: Polymer Chemistry, 9 (38), p. 4692-4705.

DOI: 10.1039/C8PY01190G

Handle: http://hdl.handle.net/1942/27167 


\title{
Sequence-Definition from Controlled Polymerization: The Next Generation of Materials
}

\author{
Jeroen De Neve, ${ }^{a, b, \dagger}$ Joris J. Haven, ${ }^{a,+}$ Lowie Maes, $^{\mathrm{b},{ }^{\dagger}}$ and Tanja Junkers ${ }^{\mathrm{a}, \mathrm{b}, *}$ \\ a. Polymer Reaction Design Group, School of Chemistry, Monash University, 19 Rainforest Walk, Clayton VIC 3800, Australia. \\ b. Institute for Materials Research, Hasselt University, Martelarenlaan 42, 3500 Hasselt, Belgium. \\ $+\quad$ These authors contributed equally. \\ * Corresponding author: tanja.junkers@monash.edu
}

ABSTRACT: An overview is given on the state-of-the-art in synthesis of sequence-controlled and sequence-defined oligomers and polymers. These materials constitute chains in which chemical information is encoded along the backbone either as multiblock copolymers (controlled) or as monodisperse sequences of single monomers (defined). Focus is placed on reversible-deactivation radical polymerization techniques as these give access to a broad variety of chemical functionalities and allow for simple and fast synthesis procedures. Sequence-controlled and -defined materials have the ability to re-shape materials chemistry due to their close resemblance to biopolymers regarding structural uniformity. The field is at the edge of being able to start exploring the distinct properties that such synthetic macromolecules may have.

\section{Introduction}

Since the advent of reversible-deactivation radical polymerizations, the development of highly complex macromolecular materials has much improved. Today, an extensive range of advanced polymer architectures is in one way or the other accessible. Nonetheless, some features of biopolymers - such as proteins and DNA cannot yet be fully reproduced. Such natural polymers bear highly selective and complex functions, fundamentally based on their primary structure, i.e. the specific order of repeating units and functionalities along their backbone. Consequently, there is a high need for specialized synthetic routes that likewise allow to achieve similar precision in synthetic materials. Inspired by this, a rapid evolution is currently occurring in polymer synthesis. A shift is witnessed toward new exciting classes of precision polymers that can be interpreted largely as information-containing macromolecules. In synthetic polymer chemistry, the versatility of monomer building blocks is endless compared to nature where DNA only uses 4 nucleotides and proteins/peptides only have a library of about 20 amino acids to encode. Yet, nature is superior in creating high-performance materials simply by controlling functional group sequences. Nowadays, polymer materials are designed with almost unlimited variations in chain length, dispersity, topology, composition and functionality. However, properties of synthetic polymers are to date still limited by the statistical nature of monomer ordering along a polymer

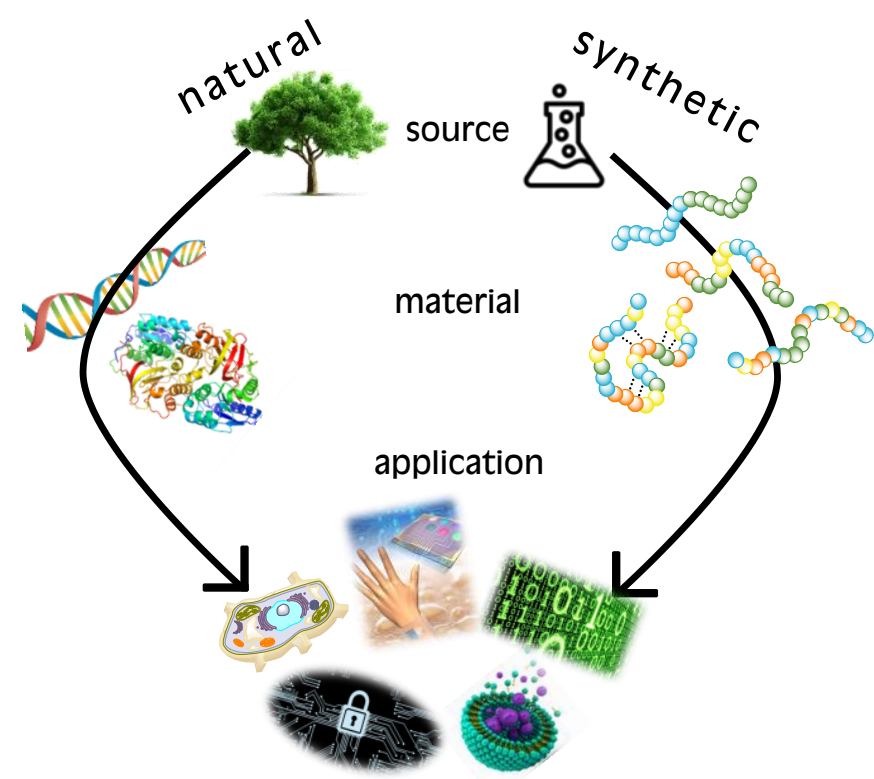

Figure 1. Comparison of natural vs synthetic polymers and their progressive convergence toward applications. Sequence-controlled and -defined materials originating from both sources can be valuable for the same future applications.

chain. Regulation of the monomer sequence in polymerizations - leading to so-called polydisperse sequence-controlled (SC) multiblock copolymers - is of high interest to bridge the above mentioned gap to biological materials in their functionality. ${ }^{1-5}$ Truly following the concept of nature, thus to synthesize monodisperse materials - so called sequence-defined (SD) polymers which are inherently different from SC materials in terms of purity and physical properties leads to a further improvement of distinct properties 
and opens new perspectives for e.g. biological and advanced functional material applications. ${ }^{6}{ }^{7}$ If properties of natural polymers such as proteins are compared with contemporary plastic materials in their specificity and versatility, the advantage of being able to mimic nature's precision in synthetic materials becomes directly evident.

Figure 1 summarizes the development of promising materials synthesized via both natural and synthetic pathways. Envisaged fields of application for these products include biomimetics, artificial peptides, molecular recognition and information storage. SD oligomers can be synthesized featuring a precise - and more importantly freely selectable - order of monomers in a monodisperse chain. In other words, such materials mimic peptides with the only difference being that in place of a peptide bond other moieties are used to connect the single building blocks. It is expected that these synthetic analogues will also be able to rearrange in precisely controlled primary, secondary or tertiary structures and can be used to perform advanced tasks such as signal transduction, selective transport or catalysis.

Many different synthesis strategies toward SC polymers have been suggested, with most of them making use of click-like chemistries or reactions as known from stepgrowth polymerizations. The reason for this is simply that addition and condensation reactions allow to add one monomer unit at a time, thus minimizing dispersity of reaction products. Iterative and orthogonal growth via non-radical chemical strategies have been reported on this matter. ${ }^{8-11}$ Although the emphasis of this review is on precision polymers via reversible-deactivation radical polymerization (RDRP) techniques, a brief overview of the most important classes of non-radical routes is presented.

Utilizing RDRP techniques, numerous multiblock copolymers have to date been obtained, mostly using acrylates or acrylamides, to encode information in the main chain. These polymers show an impressive number of blocks put in order, but are inherently of a polydisperse nature. ${ }^{12,13}$ Advances are made toward achieving monodisperse oligomers via RDRP methodologies. Such approaches require nothing less than removing or - more realistically - circumventing the statistical nature of radical chain growth. Monomers can be chosen independent of their characteristic reactivity. At the same time, a broad range of monomers is available or can be synthesized easily. It is also advantageous to use established radical chain growth techniques since they are cost-effective, inherently easy to use and scalable. Due to the radical process, oligomer growth is usually fast and not limited to one unit at a time. Furthermore, a rather chemically inert carbon-carbon backbone is formed, making SD materials from RDRP protocols considerably more stable than most counterparts made from iterative strategies, which practically always require heteroatom incorporation in the main chain. Moreover, chain extension can - after purification - be either mono- or polydisperse and a large versatility of building blocks and functionalities (from monomers to polymer endgroups) is available and tunable through e.g. postpolymerization modification. Yet, as a downside, careful purification is often required - which is, however, also the case for many other techniques - and overall product yields are on first glance low due to the separation of products out of statistical mixtures. It must be noted though that isolation of oligomers always results in a library of monodisperse compounds, which can be used for further purposes. This, in combination with the very simple synthesis procedure itself and the broad availability of cheap monomers, results in an overall very economic and efficient synthesis process. RDRP shows at the same time a high tolerance toward functional groups, allowing to polymerize polar, apolar or ionic monomers.

\section{Terminology of sequence-defined oligomers}

When discussing SD and SC materials, it is important to note on the terminology of such polymers and oligomers. To specify the number of monomer units brought into order, often the total length of the sequence is given. This approach is confusing. The number of monomers put in order is only one part of the information. Many molecules found in literature feature repeating information sequences. While such repetition may be useful in many cases from a viewpoint of the anticipated properties of such macromolecules, stating only the full length is misleading. Further, not every addition of a building block may add a specific chemical functionality, and rather serve only as spacer, or fulfill a chemical function in order to allow for monodisperse addition of a single monomer in the following step. We thus propose that the length of a sequence should be defined by its information density, hence the longest non-repeated and non-symmetrical sequence of distinct chemical functionalities within a 
molecule. A molecule of the structure A-B-C-D-E-E-D-C$B-A$ would be regarded as a pentamer just as the molecules A-S-B-S-C-S-D-S-E - where $S$ is a spacer molecule - or A-B-C-D-E. It is noteworthy to add that implementing a non-functional monomer into a sequence may serve a purpose - such as glycine in a peptide chain - in which case its introduction should be accounted for. Likewise, if oligomers feature techniquespecific end-groups, then these end-groups can only be counted toward the sequence length if the functionality is purposeful and if the functionality can be varied with said technique. Of course, for some structures gray zones exist and the identification of the smallest sequence length may not always be straightforward.

Only if the length of a sequence is counted in this way, different techniques become fully comparable. Of course, the same rules should be applied to block copolymers, where also often symmetrical polymers are formed. This leads to an increased number of blocks, but typically not to an increase in chemical information.

\section{Non-radical routes}

Various non-radical synthesis strategies are known for the design of SC and SD monodisperse macromolecules. Only a brief overview of the different classes of nonradical routes is given and the reader is referred to literature for a more detailed overview. ${ }^{14}$ As mentioned above various non-radical synthesis strategies have been suggested, with most of them making use of clicklike chemistry or reactions as known from step-growth polymerizations. ${ }^{15-17}$ The most prominent reason for this is that, during condensation reactions, dispersity of reaction products is minimized due to the addition of only one monomer unit at a time. Likewise, orthogonal and iterative growth strategies can only couple one building block at a time and in many cases require protection/deprotection strategies for the growing oligomer chain which makes it an expensive and timeconsuming approach. While some of these methods can yield products with high efficiencies, typically monomer building blocks need to be pre-synthesized, which must be taken into account when assessing the economy of reactions.

\section{Homogeneous liquid-phase synthesis of precision polymers}

Unidirectional iterative growth strategies in solution with precise control of the primary backbone structure are achieved by the Passerini three- and Ugi four- component reaction. ${ }^{18}$ To limit the amount of synthetic reaction steps, bifunctional or macrocyclic cores can be introduced for which two building blocks can be added in a single reaction step to grow an oligomer in two directions. This concept was nicely demonstrated by Zydziak et al. investigating a photochemical protocol for the generation of SD macromolecules based on orthogonal synthons. ${ }^{19}$ Another approach to limit the iterative reaction steps and rapidly reach monodisperse higher molecular weight sequences is by exponential growth strategies. Takizawa et al. synthesized a monodisperse oligo( $\varepsilon$-caprolactone) 64-mer via an exponential growth strategy to mention just one example. ${ }^{20}$ However, this method is mostly limited to palindromic or repetitive sequences and introducing versatility or side chains in the repeating units is difficult. Templated chemistry covers a polymerization process where either bio -or synthetic polymer templates are utilized to control the monomer sequence and the material properties of the final product, reviewed by ten Brummelhuis. ${ }^{21}$ Finally, Porel et al. reported the synthesis of well-defined bioactive macrocycle oligo(thioetheramides) via a one-pot acid catalyzed cascade reaction from solution (>20-mers). ${ }^{22}$

\section{Solid-phase synthesis of precision polymers}

Synthesis of SD non-natural macromolecules on a solid insoluble support, hence solid-phase synthesis, has been investigated in-depth in recent years. A great advantage of these solid-phase Merrifield methods is the simplicity of workup by filtration and washing steps. However, after cleavage of the synthesized sequence from the solid support, additional purification steps are often necessary due to incomplete cleavage or occurrence of side reactions. Although scalability of these techniques can be addressed, it requires a substantial investment and is time-consuming to start up making it only attractive for a very limited number of applications.

Martens et al. reported on thiolactone-based protecting-group-free chemistry that allows for chain elongation via a two-step orthogonal iterative method. ${ }^{23}$ Because of the orthogonality of the reactions, no protection/deprotection chemistry is involved. The thiolactone containing building blocks were pre-synthesized and multifunctional tailored side chains and backbone can be varied, as demonstrated by the synthesis of a broad variety of sequence-specific 10-mers. The reaction protocol was then transferred to an automated solid support synthesizer which 
significantly reduced the process time. A similar chemoselective $A B+C D$ iterative method was reported by Trinh et al. ${ }^{24}$ Herein, a library of eight SD oligomers whose sequence is based on a $(0,1)$ binary code - is prepared through repeating cycles of amidification and copper-assisted alkyne-azide cycloaddition reactions from a resin. The same group reported on sequences of $D P>100$ utilizing protection/deprotection chemistry on a solid support. ${ }^{25}$ Peptoid chemistry on solid supports is studied and reviewed in great detail. ${ }^{26}$ Oligo(peptoid)s are bioinspired polymeric materials that have several advantages and a similar backbone structure compared to peptides. Synthesis of oligo(peptoid)s can be performed in an automated fashion (e.g. peptide synthesizer) resulting in near monodisperse 50 -mers. ${ }^{27}$

\section{Radical routes}

\section{Sequence-controlled (SC) polymers}

RDRP techniques have given access to multiblock copolymers with predetermined block sequences. For a long time, the majority of the structures synthesized via this approach were limited to tri-, tetra- and pentablocks. ${ }^{28-31}$ Only within the last decade, the field of SC multiblock copolymers has started to further evolve and a competition between research groups emerged toward the highest number of blocks embedded into a single polymer chain. Figure 2 shows the timely evolution of the accessible sequence length for SC and SD materials synthesized via RDRP. Where pentablocks were the state-of-the-art around 2010, the situation changed quickly, and currently the longest published multiblock copolymer features 24 individual blocks in a single sequence. ${ }^{1}$ A similar trend is observed for SD oligomers, with sequences shifting from dimers in 2008 to currently decamers, solely created by RDRP methods

Race toward high-order multiblock copolymers via RDRP. An early example for the synthesis of high-order multiblock copolymers was given by Gemici et al. who obtained multiblock copolymers via reversible additionfragmentation chain transfer (RAFT) polymerization. Telechelic ABA-type triblock copolymers were synthesized and reduced into $\alpha, \omega$-functional thiols. Subsequent oxidation of the functional thiols resulted in multiblock copolymers with disulfide bridge formation between the telechelic ABA-type blocks. ${ }^{32}$ While such approach delivers polymers with a very high number of blocks, only an alternation of two blocks could be achieved.
Over the years, the synthesis of materials with true monomer sequences became accessible via constant refinement of reaction conditions. In order to add multiple blocks to a living chain, one at a time, polymerization methods are required that allow for a high end-group fidelity (high livingness) of polymer chains in the multistep process. At the same time, high monomer conversions must be obtained to avoid tedious purification steps after every block extension. The techniques used to fulfill these requirements are not different from the classical RDRP methods. In classical recipes for RDRP, livingness of chains is dramatically reduced when reaching high monomer conversions as less monomer is available for propagation, eventually favoring termination events. Nevertheless, this effect can be overcome if conditions are chosen carefully. In this sense, the further development of SC was not triggered by novel polymerization techniques, but by a deeper understanding of the underlying polymerization kinetics and hence optimization of methods close to perfection. $\mathrm{Cu}(0)$-mediated radical polymerization (single electron transfer living radical polymerization, SET-LRP) was the first method to demonstrate the potential of optimized reaction conditions. ${ }^{33}$ Soeriyadi et al. used optimized SET-LRP and derived octa- and decablock copolymers consisting of different acrylate monomers. ${ }^{34}$ The polymerization was performed at ambient temperature and full monomer conversion (24h per block) was reached after each block extension. Therefore, purification between subsequent polymerization steps was unnecessary. The synthesized block copolymers showed high end-group fidelity (80-90 \%) after each step and dispersities between 1.20-1.27 were observed. The same procedure was used for the synthesis of multiblock star copolymers via a core-first approach and optimized for the synthesis of higher molecular weight multiblock copolymers ( $D P=100$ for each block). ${ }^{35,36}$ While first examples were still limited in the end-group purities that could be obtained, these papers certainly triggered further developments (see Figure 3 for an overview).

Inspired by these initial findings, subsequent research broadened the scope of the approach. This led for example to the implementation of other monomer families such as acrylamides in multiblock copolymers by employing a PEG-macroinitiator in aqueous media at reduced temperatures. ${ }^{37-39}$ In further adaptations, SETLRP was replaced by photo-induced atom transfer 

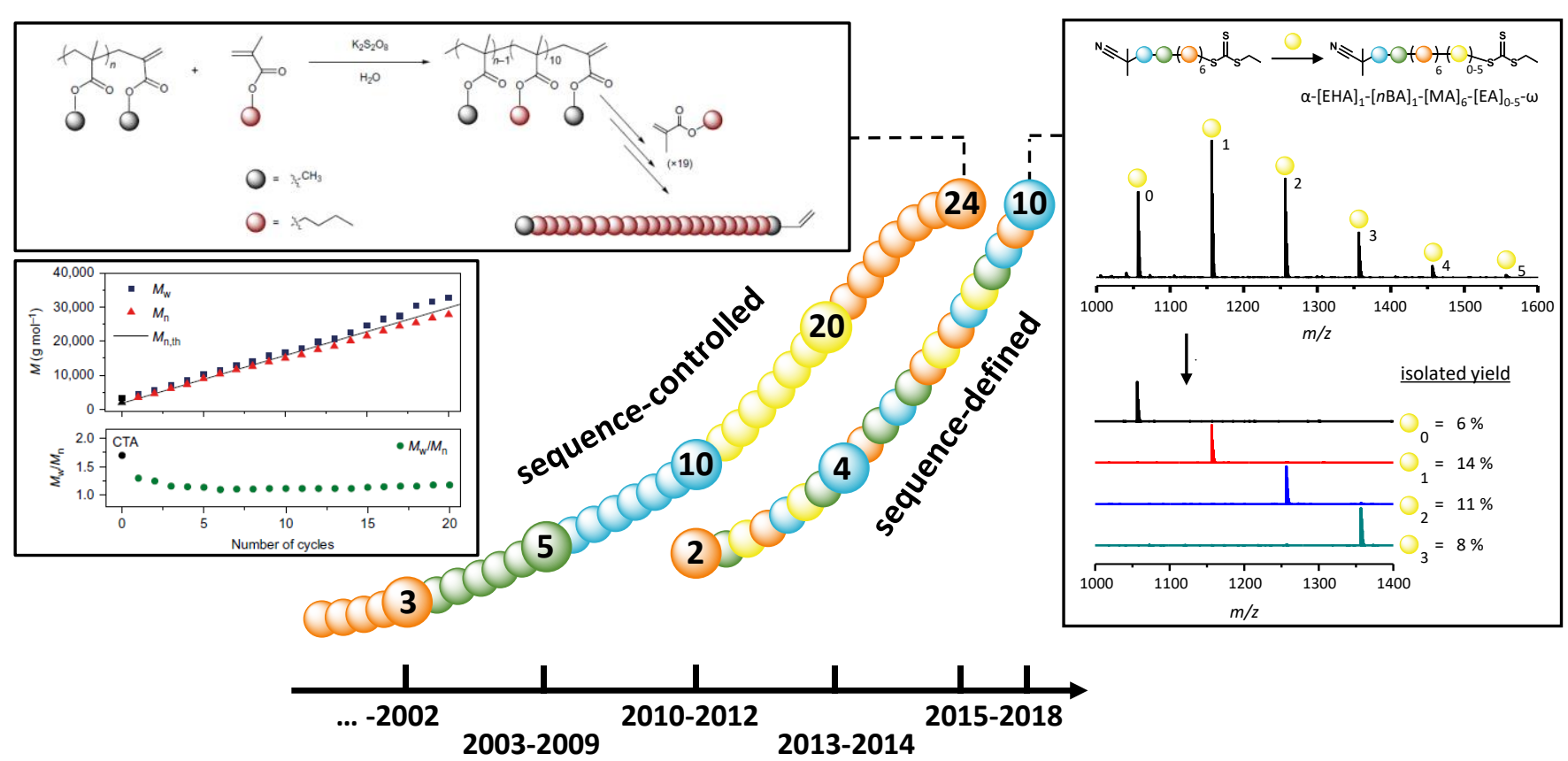

Figure 2. Evolution of sequence length in sequence-controlled and-defined materials via reversible-deactivation radical polymerization. The curves represent the number of blocks in sequence-controlled (SC) polymers or number of monomer units in sequence-defined (SD) polymers, respectively, as evolution in time. Schemes on the left and right hand side of the curves resemble the synthesis procedures used to obtain the current record holders for the longest sequence in SC and SD materials respectively. Displayed sequence lengths originate from the following references: SC 3[31]; SC 5[30]; SC 10[34]; SC 20[13]; SC 24[1]; SD 2[84]; SD 4[92]; SD 10[91]. Insert figures reproduced with permission from Ref ${ }^{91}$ and Ref ${ }^{1}$. Copyright (c) 2017 American Chemical Society and (c) 2016 Nature Chemistry.

radical polymerization (photoATRP) for the synthesis of SC polymers. ${ }^{40}$ PhotoATRP is known to be very efficient in terms of livingness and also allows to achieve practically full conversion without significant loss of end-groups. After sequential block additions of various acrylates through photoATRP, an undecablock was obtained with a narrow dispersity $(\Theta<1.2)$ and similar length for each block $(D P=3)$. Also higher molecular weight multiblock copolymers are within reach and further optimization gave rise to $\alpha, \omega$-telechelic SC polymers up to a tricosablock $(\oplus=1.18)$ using a bifunctional initiator (with a sequence length of 12) thereby proving the versatility of this approach. ${ }^{41}$ This was confirmed by the work of Junkers and coworkers, who synthesized decablock copolymers from photoATRP as well as star polymers, stretching over 8 block additions from a 4-arm star core initiator. ${ }^{42,43}$

Initially, only reversible termination techniques such as SET-LRP or ATRP were thought to be capable of producing SC polymers, as the effective suppression of termination gave the foundation to reach high conversions and livingness concomitantly. However, shortly after the development of these procedures Gody et al. demonstrated that also degenerative transfer polymerization such as RAFT polymerization is able to fulfil the same criteria (Figure 3). This was undeniably proven by synthesis of icosablock copolymers. $^{13}$ By using a one-pot iterative RAFT polymerization procedure, hexa-, dodeca- and icosablock copolymers were synthesized with each block reaching near full conversion in $24 \mathrm{~h}$ (hexa -and dodecablock copolymer) and $2 \mathrm{~h}$ (icosablock copolymer) per block. This icosablock consisted of alternating blocks of 4-acryloylmorpholine and $\mathrm{N}, \mathrm{N}$-diethyl acrylamide with a $D P=3$ and was the highest achieved SC polymer up until then. The overall dispersities of the final multiblock copolymers range from 1.36-1.57 and

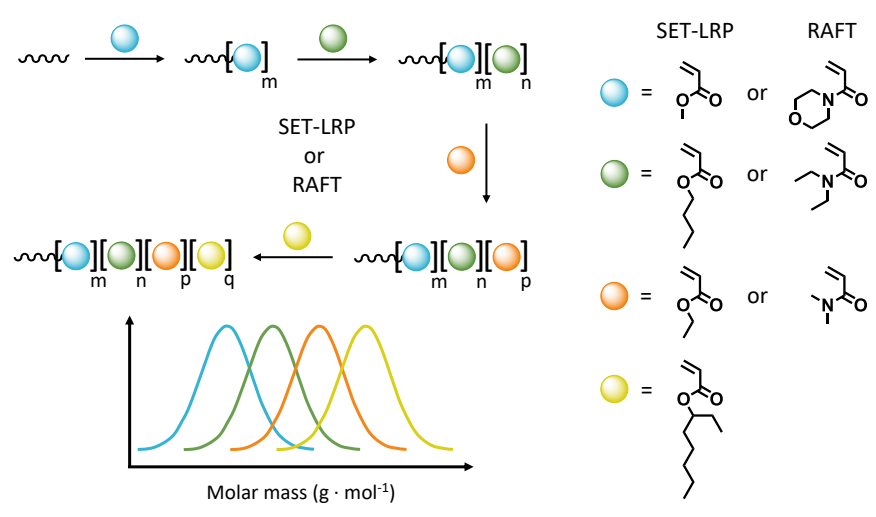

Figure 3. Creation of sequence-controlled multiblock copolymers. Simplified scheme exemplifying the synthesis strategy of multiblock sequences through subsequent single-electron transfer living radical polymerization (SET-LRP) or reversible addition-fragmentation chain transfer (RAFT) polymerization. 
continuously increase (from 1.2 to 1.6) after each block extension. With further optimization, simulation and modelling it was possible to synthesize multiblocks in a much faster way while still maintaining low dispersities and compatibility with different molecular weights of blocks even in aqueous media. ${ }^{5,44-46}$ The reason RAFT polymerization could be employed for such synthesis targets is the careful fine-tuning of the initiator content. While RAFT polymerization relies on exogenous radical initiation, the number of dead chains is directly linked to the number of initiator molecules present at the start of the reaction. While RAFT polymerization relies on exogenous radical initiation, the number of dead chains is directly linked to the number of initiator radicals generated during the polymerization. If reaction conditions are chosen carefully, $99 \%$ livingness can be reached at completion of the reaction. ${ }^{44}$ This approach has shown to be very powerful and is even improved by the newer development of RAFT iniferter polymerization, ${ }^{47}$ yet requires fine-tuning of conditions and is only achievable with monomers of sufficient propagation rates in order to avoid that the initiator depletes before high monomer conversions are reached. ${ }^{48}$

Harrisson et al. used the RAFT polymerization SC system to demonstrate an interesting feature of this special type of polymers. ${ }^{49}$ Each block extension creates a Poisson distribution of chains per segment. Interestingly, when short blocks of 3 or less monomers are targeted, a significant portion of chains in the mixture is not extended at all and exhibits zero monomer insertions. Thus, in consequence, information is lost. From this observation a recommendation is drawn that block extensions must be of considerable length (at least 5-10 monomers) in order to guarantee that the desired information is stored in the polymer. This is somewhat in conflict with most optimization protocols of polymerizations, as shorter blocks generally allow for faster polymerizations, and hence are needed to fulfil the stringent kinetic criteria for successful sequence-control. This comes on top of the fact that especially RAFT polymerization leads to a loss of sequence information by the reinitiation nature of each block extension; the initiator concentration does not only contribute to a loss of livingness, but also starts fresh homopolymers during each block extension, leading to complex mixtures of block copolymers with various sequence lengths at the end of the synthesis cycle. ${ }^{50}$ It is for this reason that at the moment less publications focus on longer sequences, but rather at the creation of polymers where the block length is significantly larger for each block.

Searching for more functionality. In parallel with the race for the highest-order multiblock copolymers also SC materials with functionalities at specific sites along the backbone were investigated. In such case the polymer consists primarily of a single monomer type, where specific functionalities are added along the chain only in certain locations. An interesting approach to obtain such copolymers with specific functional sites is applied by Pfeifer et $a l^{51}$ The principle of this technique is based on the controlled sequential addition of $N$ substituted maleimides during the ATRP of styrene (see Figure 4). These comonomers have a very low tendency to homopolymerize and are favoured for crosspropagation with styrene. By addition of these comonomers at specific times during the reversibledeactivation radical polymerization of styrene a copolymer with functionalities at distinct sites along the backbone can be obtained. This approach was thoroughly investigated and specific synthesized $N$ substituted maleimides were used by changing the Rgroup and using styrene derivatives. Automated protocols and biomolecules like sugars were also implemented to prove the broad applicability of this technique. ${ }^{52-54}$ Furthermore, this method allows for the synthesis of a SC polymer with folding sites finding possible application in the field of biomimetics. The underlying principle can also be extended to other reversible-deactivation radical polymerization mechanisms like nitroxide-mediated radical polymerization (NMP). ${ }^{55}$ The combined use of $N$ substituted maleimides and styrene was the inspiration for other researchers to experiment with bio-inspired monomers and dig into the possible characteristics of these SC polymers. ${ }^{16,56}$

\section{Recent developments in sequence-controlled} synthesis procedures. In the last three years, the characteristics of SC materials became more and more clear. The race for the highest multiblock via one-pot synthesis strategies is stagnating and the focus shifts more and more to the synthesis of well-defined polymers via "greener" methods with growing interest in application for the biochemical and biomedical field. However, investigations are still ongoing toward better methods to synthesize high-order multiblocks. Recently, Engelis et al. have set the record of the highest-order SC polymer by using sulfur-free RAFT emulsion polymerization. Multiblock copolymers up to 24 blocks 

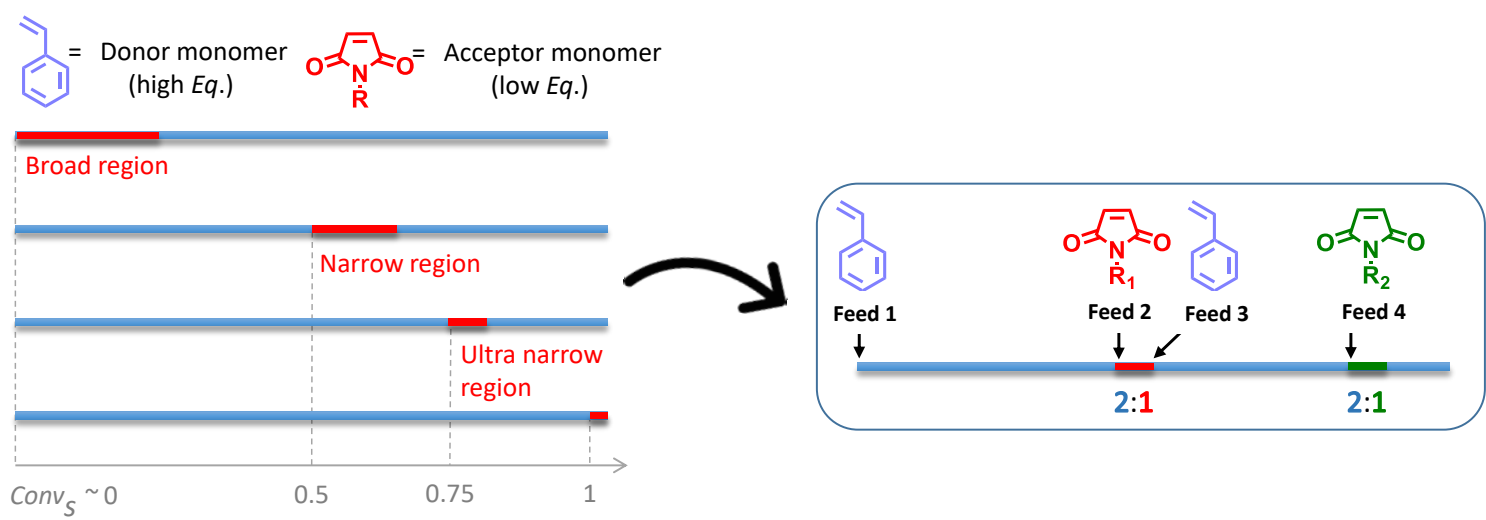

Figure 4. Sequence-controlled polymers via a precise insertion strategy. Precise insertions of an acceptor monomer, like $\mathrm{N}$-substituted maleimides, into the main chain of a styrene donor monomer results in a copolymer with well-defined segments. The time on which the acceptor monomer is inserted determines the distribution of the segment. Combination of different $\mathrm{N}$-substituted maleimides leads to a SC polymer with varying functionalities. Adapted with permission from Ref ${ }^{52}$. Copyright @ 2007 American Chemical Society.

were synthesized using methacrylic monomers. ${ }^{1}$ Again, the applicability of this method was demonstrated by using low and high molecular weight blocks linked together in a sequential addition fashion. Further optimization of this approach resulted in the synthesis of comb-like diblock polymers and the scope was expanded by using four different macro chain transfer agents. ${ }^{57,58}$ Other one-pot synthesis approaches focus on the use of hydrophobic/hydrophilic monomers or exploit the donor/acceptor interaction of styrene/ $\mathrm{N}$ substituted maleimides to synthesize new types of SC polymers. ${ }^{59-61} \mathrm{Ji}$ et al. adapted the principle by using furan protected maleimides. ${ }^{17} \mathrm{~A}$ shift to a more biochemical orientation of the SC field is clearly visible in types of monomers that are used and the methods that are applied. Lavilla et al. used protected $N$ carboxyanhydrides for the synthesis of SC glycopolypeptides which have a binding affinity for different lectins depending on the sequence of the peptide blocks. ${ }^{62}$ These SC polymers - with peptide functionalities and structures similar to proteins - can be synthesized by various techniques such as NMP and photo-induced electron/energy transfer (PET)-RAFT polymerization and have the possibility to form bioconjugates with natural biomolecules or even have an antimicrobial activity. ${ }^{63-65}$ Recently, other interesting pathways have been suggested for the synthesis of these precision polymers. The possibility of using flow chemistry to synthesize these type of polymers was shown by Baeten et al. to scale up polymerizations. ${ }^{66,67}$ Kuroki et al. demonstrated that the synthesis procedure can also be expanded to a looped flow reactor. ${ }^{68}$ In another approach a DNA synthesizer was programmed for the automated synthesis of diblocks via photoATRP. "69 "Green" methods to synthesize ABCBA multiblock copolymers were introduced via sulfur-free
RAFT emulsion polymerization without surfactant or organic solvent. $^{70}$

\section{Sequence-defined (SD) polymers}

As described above, SC multiblock copolymers are readily accessible via RDRP with remarkable yield and end-group fidelity. Although high-order structures can be synthesized this way, the block copolymers are still of a polydisperse nature and therefore not fully SD. The distribution of monomer locations along a polymer chain is influenced by the statistical nature of the chain growth polymerization and resulting products obey a Poisson or Beta distribution. ${ }^{49}$ This inherent distribution affords limited control over the final structure which could lead people to conclude that precise control over the absolute position of a monomer is not achievable via radical chain growth polymerizations. However, various strategies have been developed to circumvent this issue and the creation of SD materials through RDRP pathways is now a mature research field as will be discussed below.

Alternating sequences. As a first step in the direction of SD materials, alternating sequences were created via RDRP. ${ }^{71}$ Although still SC in nature, the use of nonradical methods to synthesize building blocks and subsequent polymerization already highlights an interesting aspect of the technique. In one reaction step, the same structural element can be incorporated multiple times into a polymer chain. Hibi et al. designed template monomers carrying two types of polymerizable alkene functionalities which were placed side by side on a rigid naphthalene scaffold. ${ }^{72}$ Such a setup allows the formation of linear controlled polymers with highly alternating sequences of for example acrylate (A) and methacrylate (B) through selective 
2)
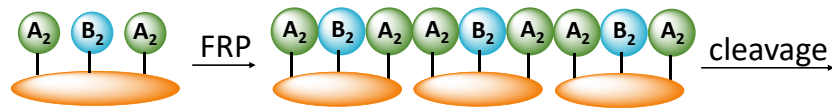
$\mathrm{A}_{2} \mathrm{~B}_{2} \mathrm{~A}_{2} \mathrm{~A}_{2} \mathrm{~B}_{2} \mathrm{~A}_{2} \mathrm{~A}_{2} \mathrm{~B}_{2} \mathrm{~A}_{2}$

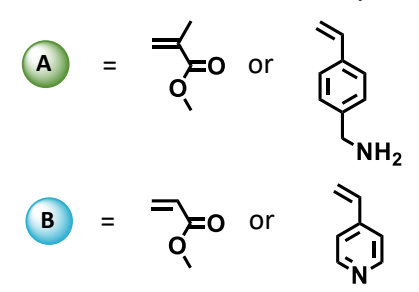

Figure 5. Alternating sequences. Synthesis of alternating polymer chains starting from a monomer bearing scaffold followed by polymerization and cleavage of the starting template. RDRP $=$ reversible-deactivation radical polymerization, FRP $=$ free radical polymerization.

intramolecular radical propagation (Figure 5). However, the technique requires diluted conditions and is prone to solvent effects with conversions over $90 \%$ reached only in anisole and 1,2-dichloroethane. Apart from the high alternating triad sequence ( $A B A)$, an unusual heterotactic-rich tacticity can be observed which is attributed to the naphthalene scaffold.

In subsequent work, a more elaborate alternating sequence was created by switching from an $A B$-carrying naphthalene scaffold to an ABA-carrying palladium scaffold (Figure 5). ${ }^{73}$ This allows incorporation of a greater variety of functional groups. Nevertheless, aromatic ring structures are required in the three monomeric units incorporated in the scaffold to allow $\pi-\pi$ stacking interactions aligning the array for consecutive polymerization. In order to obtain a regular sequence, free rotation of the outer elements $(A)$ and subsequent crosslinking needs to be suppressed by utilizing a bulky fluoroalcohol solvent. Also, molecular mobility within the monomer must be further reduced by performing the polymerizations below $-5^{\circ} \mathrm{C}$.

Radical polyaddition using pre-synthesized building blocks has also been explored. ${ }^{71}$ Alternating vinyl copolymers bearing vinyl chloride (A), styrene (B) and acrylate (C) units were synthesized. However, even after extensive reaction optimization, only moderate molecular weights are achieved. Although cyclic byproducts resulting from intramolecular cyclization of the $A B C$ scaffolds are inevitable, less side-reactions were observed by increasing bulkiness of the ester substituent and could be even further reduced by employing ABCC-type monomers. Given the constraints of the above systems, it is not possible to create more well-defined functional synthetic macromolecules.

Template-assisted polymerization systems. Further efforts in the development of SD materials were inspired by template-assisted procedures as seen in the natural synthesis of biological macromolecules. ${ }^{74-76}$ This approach was adapted by introducing a templatebearing initiator in the form of a hairpin-shaped rigid framework for subsequent metal-catalyzed radical addition (Figure 6). ${ }^{74}$ The specific interaction between the template amine and the acid in methacrylic acid (MAA) permits the predominant synthesis of 1:1 adducts with the halide being consumed at almost the same rate as MAA. Furthermore, this approach enhances the competitive radical addition of MAA over methyl methacrylate by a factor of 10 . Due to the ionic nature of the interactions, solvent polarity is crucial for the recognition susceptibility with selective single monomer addition also being sensitive toward substrate concentrations. Specific mono-addition is favoured at concentrations under $50 \mathrm{mM}$ in less polar solvents such as toluene.

In an attempt to further optimize the system, the amine template moiety was substituted for a lariat crown ether function. ${ }^{75}$ This alteration should allow the recognition of ionic monomers according to their cation size. Selective insertion of sodium methacrylate over a much larger ammonium cation derivative can in this way be achieved (Figure 6). The template effect increases with decreasing reaction temperature, but a full selectivity for sodium methacrylate seems out of reach. Although both above methods showcase the specific insertion of a monomer unit, the approach seems rather tedious with little room for further development toward longer and more complex defined structures.

An even closer resemblance to nature's synthesis strategy during DNA translation focuses on radical cyclization with orthogonally cleavable and renewable bonds. ${ }^{76}$ The initiator-monomer (inimer) template in this process carries both a radical-generating site and an 


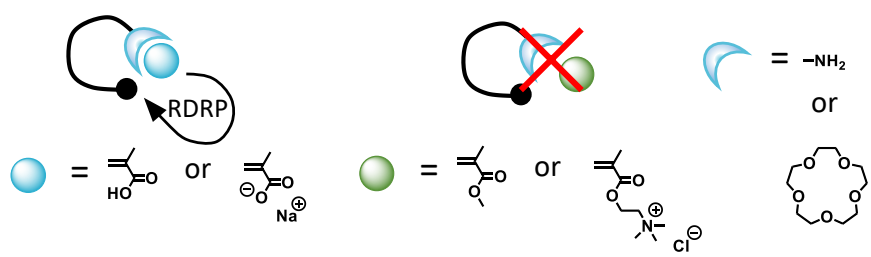

Figure 6. Template-assisted monomer addition. Addition of a suitable monomer through competitive metal-catalyzed polymerization - recognized by the template moiety - resulting in 1:1 adducts. RDRP = reversible-deactivation radical polymerization.

alkene leading to a repetitive and iterative process of single monomer addition through cyclization, bond cleavage and regeneration. In order for this procedure to succeed, the metal catalyst needs to be carefully selected and diluted reaction conditions are applied for selective radical formation and cyclization without further chain growth. Apart from that, orthogonal bond cleavage and quantitative regeneration with the possibility for side-chain functionalization is crucial to be able to repeat the process. For every additional ringclosure, the conditions need to be tuned by altering solvent, temperature and catalyst amount. A variety of side chains can in principle be implemented upon cleaving the macrocycle, however, care must be taken not to build-in moieties that interfere with subsequent additions. In the end, a total of two radical additions with a conversion $>90 \%$ yielded a SD trimer, but synthesis of the starting inimer was limited to $30 \%$ conversion. Overall an ingenious, iterative method for the template-assisted synthesis of well-defined sequences through radical addition of a single monomer at a time was designed. However, the application potential is rather limited due to the complexity of the whole approach.

Radical addition reactions. The synthesis of controlled sequences of vinyl monomers can also be achieved through radical single unit addition with subsequent reactivation of the chain end. ${ }^{77,78} \mathrm{~A}$ first example of this approach was shown by Tong et al. who employed an atom transfer radical addition (ATRA) based strategy. ${ }^{77}$ An allyl alcohol is added to a bromine carrying chain end after which the hydroxymethyl residue is oxidized to a carboxylic acid. Subsequently, a desired side group can be introduced via esterification creating an active chain end to repeat the radical addition cycle. Although seemingly straightforward, the iterative cycle of monomer addition, pendant oxidation and esterification does entail a distinct drawback. Chain transfer side reactions occur leading to a $20 \%$ loss of bromine end-groups upon each allyl alcohol addition. Further conversion of the alcohol to a carboxylic acid

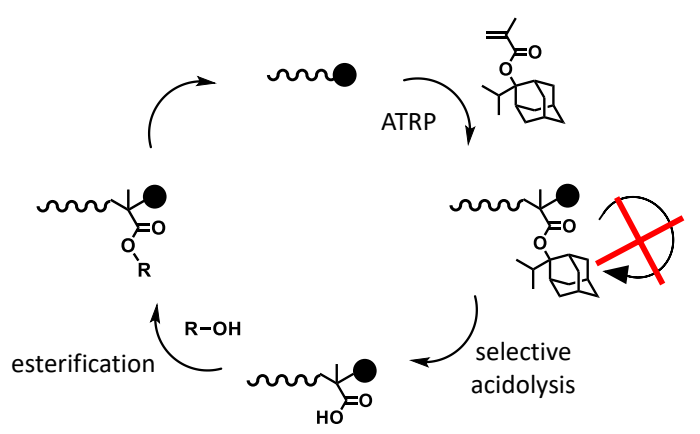

Figure 7. Radical addition with a bulky monomer. Adding one monomer at a time via radical addition is made possible through a monomer carrying a bulky and convertible pendant. Subsequent additions become available through post-modification steps. ATRP = atom transfer radical polymerization. Adapted with permission from Ref ${ }^{79}$. Copyright (C) 2016 American Chemical Society.

group through an Anelli oxidation procedure and following esterification proves effective. While monoaddition and chemical transformation have been executed, iteration of the three-step cycle has yet to be shown

Lately, a different concept toward controlling the sequence for vinyl polymers was established, relying on monomers carrying a bulky and convertible pendant group. $^{78}$ The exceptional bulkiness of an alkyl methacrylate bearing both adamantyl and isopropyl groups is able to control single unit addition during ATRP. While the double bond is reactive enough for a first addition, the steric hindrance originating from the bulky sidechain prevents further propagation as soon as one unit is built in (Figure 7). Through acidolysis and subsequent esterification, the side chain functionality can be modified and the polymer chain is reactivated to allow following additions, creating a well-defined methacrylate sequence. Acidolysis proceeds quantitatively and various alcohols can be used for the esterification although only modest yields are reached for this step. A total of three addition cycles have been completed with only minor alterations to the reaction temperature in the second and third phase. Both discussed approaches carry an unmistakable value for the creation of short SD structures. Nevertheless, their elaborateness with the need for multiple intermediate reactions - before achieving addition of a next monomeric unit - is a disadvantage when trying to access longer chains.

Single unit monomer insertion (SUMI). A new era for the synthesis of SD polymers emerged when the kinetics of RAFT polymerizations were further studied and unraveled..$^{79-86}$ The insights gathered from these investigations eventually led to what is now known as a 
single unit monomer insertion (SUMI). Already in 1988, Delduc et al. laid the first foundations for the trapping of radicals stemming from xanthates via a radical chain process using less-activated monomers (LAM). ${ }^{79}$ The executed photoreaction procedure effectively incorporates one unit into the RAFT agent. ${ }^{80}$ Under slightly diluted conditions and with an excess of RAFT agent, predominantly single insertion products - with only minor formation of oligomers - are observed. The unwanted oligomerization stems from enhanced stabilization of an intermediate radical thereby promoting back-fragmentation.

A study of the early-stage kinetics of RAFT polymerizations, led McLeary et al. to the realization that there is a selective initialization during the initial consumption of RAFT agent, not to be mistaken with inhibition. ${ }^{81} \mathrm{~A}$ key factor in this process is a selective fragmentation of intermediate radicals toward radicals that have not yet undergone propagation. Furthermore, also addition of radicals - with a $D P$ of at least one - to the RAFT agent being faster than to monomer is of prime importance. ${ }^{82}$ The rate coefficients for addition and fragmentation depend on the $D P$ and the type of propagating chains. Therefore, large variations can be observed during the first few monomer additions. As a result, no longer oligomers are observed before all RAFT agent is converted to the single monomer adduct. This behaviour is no longer noticed for subsequent monomer insertions after the initialization phase.

Chen et al. showed the implementation of the SUMI strategy making use of more-activated monomers (MAM) in the design of light harvesting macromolecules. ${ }^{83}$ By deliberately keeping the molar ratio of monomer to RAFT agent at 1:1, single chromophore units could be introduced into the RAFT agent. Further insight into the SUMI process was established by Moad and coworkers who were the first to synthesize both a trithiocarbonate and dithiobenzoate macroRAFT agent via two successive single unit MAM insertions of styrene and NIPAM respectively. ${ }^{84,85}$ Crucial for an optimized yield of the desired product is a careful selection of initiator, monomer, RAFT agent and reaction time. The transfer constant of the RAFT agent needs to be adequately high so that per activation cycle less than one monomer unit is added. Apart from that, the addition rate of the radical to the monomer should outperform further propagation meaning that reinitiation occurs as efficiently as possible. The sequence that can be achieved is hence outlined by kinetic constraints and cannot be chosen freely. Furthermore, a low ratio of monomer to RAFT agent favors single monomer insertion while extended reaction times lead to a reduction in the yield with the final desired macroRAFT agent being obtained in $35 \%$ isolated yield. Therefore, stopping the reaction before full monomer conversion is advised.

Photo-activated SUMI. Photo-induced radical reactions for the development of controlled polymer architectures found their way into the field of SD polymers when the group of Boyer and coworkers used PET-RAFT polymerizations for the design of SUMI structures. $87-89$ Incorporation of various monomer families like acrylates, acrylamides and styrenes via the SUMI strategy proved successful. ${ }^{87}$ The PET process effectively activates and deactivates the RAFT agent in the presence of trace amounts of photoredox catalyst. The use of photoredox catalyst is beneficial as it avoids other initiator species and consequently also the formation of initiator-derived by-products. However, even the use of excess monomer results in reaction times around $20 \mathrm{~h}$. Isolated yields of the SUMI products of acrylates, acrylamides and styrene approach $90 \%$ while SUMI of methacrylates is unsuccessful through this method. In this last case, repeated activations without selectivity lead to the formation of an oligomeric product. Although this approach is elegant to insert a single species into a RAFT agent for a number of monomer families, no longer defined sequences could be obtained at that point using the same system of RAFT agent and photoredox catalyst as additional controlled insertions were not possible under identical conditions.

A further refinement of the technique gave rise to SD trimers. ${ }^{88}$ The discrete oligomers can be obtained via sequential PET-RAFT SUMI reactions through a

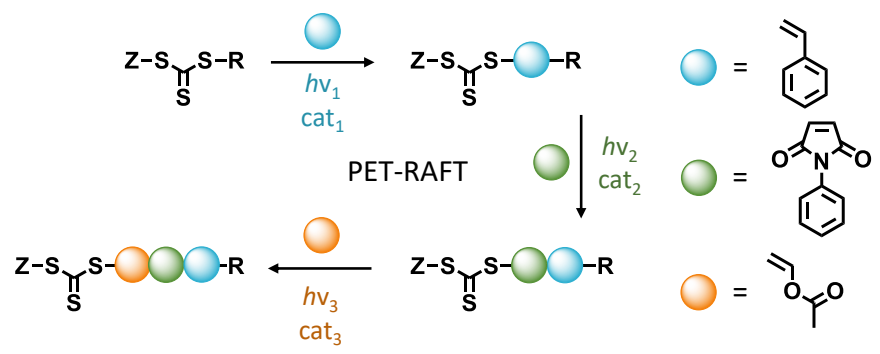

Figure 8. Photo-activated single unit monomer insertion. Design of a SD trimer by careful selection of monomer, photoredox catalyst, control agent and light source wavelength. PET-RAFT = photoinduced electron/energy transfer reversible addition-fragmentation chain transfer, cat = photoredox catalyst. Adapted with permission from Ref88. Copyright 2016 Wiley-VCH Verlag GmbH \& Co. 
meticulous selection of RAFT agent, photoredox catalyst and monomer (Figure 8). The RAFT agent used, has a high transfer constant toward a range of monomers such as methacrylates and styrenes whilst having a high radical to monomer addition rate, specifically in the case of styrene. At room temperature the propagation rate is lowered, thereby further limiting the possibility of dimer formation. Nevertheless, it must be considered that upon light irradiation a local increase in temperature will also be observed. During incorporation of the third and final monomer, around $10 \%$ of uncontrolled multiple insertion products are formed, bringing in the need for careful purification to obtain the SUMI product. With an optimized configuration, the overall yield is $75 \%$ after three reactions. The presence of RAFT end-group functionalities allows easy post-oligomerization modification of these well-defined sequences. Examples include the formation of regulated hexamers through a DCC coupling and the synthesis of a graft copolymer with uniform SD grafts.

$\mathrm{Fu}$ et al. later modified the strategy with the implementation of intermediate thiol-ene and esterification reactions. ${ }^{89}$ The scope here is expanded toward catalyst-free RAFT SUMI with faster propagating monomers. Due to this less favourable kinetic parameter, the resulting products contain a mixture of multi-monomer adducts with up to three monomers inserted, making purification via column chromatography inevitable. After purification, the RAFT end-group undergoes aminolysis and an in-situ thiolene Michael addition reaction with an alcohol bearing acrylate. Subsequent esterification of the hydroxyl group yields a new macroRAFT agent that can undergo consecutive cycles of SUMI, aminolysis, thiol-ene and esterification. This approach is exploited to create a SD trimer with an overall yield of $16.5 \%$ calculated from the initial RAFT agent. Claims of a higher-order SD structure can be made since additional, selectable acrylates are inserted during the Michael addition. However, these monomers are built into the oligomer backbone with the sole purpose of adding a next RAFT agent to them instead of having a pending functional side chain. Overall the research shows that PET-RAFT polymerization is a viable option for the creation of welldefined sequences with various monomer families when both RAFT agent, monomer and catalyst are carefully selected and attuned to one another. Recently, Aerts et al. explored the influence of irradiation wavelength, intensity and reaction temperature to perform a selective SUMI of $\mathrm{N}, \mathrm{N}$-dimethylacrylamide
(DMAm) via light-induced RAFT polymerization in aqueous solution..$^{90}$ The specificity for SUMI over formation of longer oligomers is higher for longer irradiation wavelengths. Also, SUMI appears more rapid and more selective at higher temperatures $\left(65^{\circ} \mathrm{C}\right.$ vs ambient).

From optimized single to multiple unit insertion. Based on the early principles of SUMI reactions, the boundaries of SD structures have been pushed through the optimization of synthesis and purification protocols with the use of on-line monitoring, kinetic simulations and most notably semi-automated chromatography separation. (Figure 9)..$^{91-96}$

In a first approach, well-defined structures are designed with up to four distinct acrylate monomer insertions via the RAFT polymerization technique. ${ }^{92}$ Fast polymerizations (10 minutes) were performed showing $>99 \%$ RAFT end-group retention. To cope with the statistical nature of a radical polymerization reaction, a purification step after each insertion will always be required to obtain the desired SUMI material. Preparative recycling size-exclusion chromatography (recSEC) is found to allow facile and automated purification to obtain monodisperse SD materials. RecSEC operates by running the mobile phase back (in cycles) over the columns until all SUMIs are separated. It must be noted that separation gets more difficult the more monomer units are added to the growing oligomer chain as the hydrodynamic volume does not

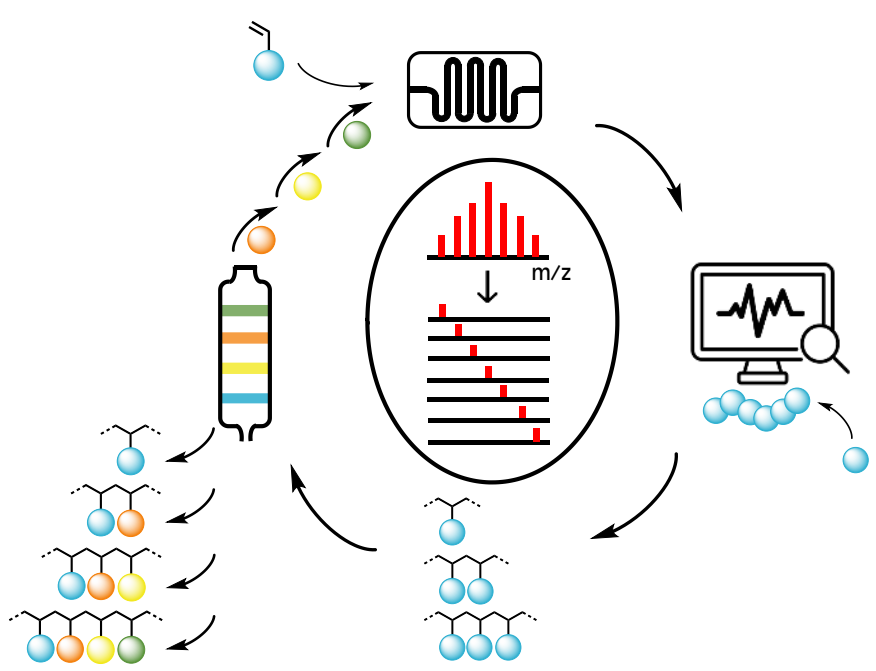

Figure 9. Synthesis of sequence-defined structures in combination with chromatography separation. Process showing the various steps in an optimized synthesis protocol to reach well-defined architectures with microflow reversible-deactivation radical polymerization coupled to on-line screening to monitor the production of various insertion products followed by automated purification yielding SD monodisperse polymers. 
linearly increase with chain length. Where the first insertion still results in a respectable yield of $55 \%$ (which is close to the kinetic maximum achievable in the crude yield), yields of $15 \%$ are obtained for tetramers. To further optimize the SUMI process, a technique for the continuous on-line monitoring via electrospray ionization mass spectrometry (ESI-MS) microreactor coupling has subsequently been established..$^{93}$ The value of real-time data stemming from on-line analysis of chemical processes is unmatched for rapid kinetic screening and thus the efficient optimization of chemical reactions and specific product patterns from SUMI reactions. Yields for the single insertion product were optimized by screening reaction temperature, residence time and reagent ratios. Only trace amounts of material and minimal time are required via this technique. Optimized reaction conditions were easily scalable (100x) from a micro- to miliflow reactor.

A concurrent kinetic screening and modelling by Haven et al. provided more insight into the process of SUMI reactions. ${ }^{94}$ More specifically, kinetic modelling is employed to examine reaction efficiencies of monomer insertions via RAFT polymerization and explore which parameters have a beneficial or adverse influence on the reaction outcome. Rather unexpectedly, key parameters such as radical flux, termination and RAFT addition rate coefficients or monomer concentration at a fixed ratio show to have only limited influence on the outcome of the simulation. However, the propagation rate exhibits a strong chain length dependency with dramatic rate variations for the first few steps. It thereby has a major impact on individual reaction yields that show to decrease for each subsequent insertion. These results closely resemble experimental observations, demonstrating the clear role of chain length effects in SUMI reactions. From these insights, some recommendations for reaction optimization can hence be postulated. RAFT agent and monomer concentrations can be chosen freely while initiator concentrations and temperature can be picked according to the desired reaction time. An annotation needs to be made that side reactions such as backbiting and $\beta$-scission are more pronounced at elevated temperatures. At the same time, initiator concentrations have no significant influence on the reaction yield, unless extreme conditions are considered. Insertion of multiple units - resulting in a reduced SUMI product yield - does not have a marked effect when the monomer to RAFT agent ratio is kept below 10:1. Hence, to increase the overall polymerization rate, higher monomer equivalents instead of equimolar conditions are recommended for SUMI reactions. The herein described product distributions are an immediate effect of the individual rate coefficients rather than the employed control methodology. Therefore, the results should be well transferable to other RDRP techniques.

To expand the scope of SUMI reactions, the insights gathered from previous studies were combined with photo-induced copper-mediated radical polymerization (photoCMP). ${ }^{7}$ Consecutive SUMI reactions are performed with purification of the product mixtures through recSEC to obtain a SD acrylate pentamer. The advantage of photoCMP is that it renders high polymerization rates under mild reaction conditions, thereby limiting undesirable side reactions. To closely monitor the reaction progress, these photo-induced SUMI reactions are followed by in situ Fourier-transform infrared spectroscopy. Care must be taken to avoid any residues from chlorinated solvents as they will undergo undesired halogen-exchange side reactions with subsequent chlorine-terminated materials being inactive for following photoCMP reactions. In order to realize an efficient separation of the various insertion products and obtain the highest possible reaction yields, intermediate purification steps are strongly recommended over a single tedious purification step of the final crude mixture. Important to clarify is that the different oligomers formed in each SUMI step may be of equal relevance. A library of SD products is obtained from every insertion reaction. In that respect, one must also bear in mind the individual yields of all isolated oligomers next to the overall yield of a SUMI reaction when evaluating the efficiency of a SUMI process. Compared to RAFT-based SUMI reactions, photoCMP results in comparable reaction yields, as predicted by previous simulations, and is equally efficient in synthesis thus exemplifying that practically any RDRP method can be used for generating SUMI products.

In the most recent study, a monodisperse decamer was obtained which is the to-date longest SD oligomer structure reported via RDRP. ${ }^{91}$ The approach embraces the fact that when using RDRP techniques, oligomer growth is not limited to one unit at a time. Multiple unit monomer insertion (MUMI) is the inherent power of a RDRP system for the synthesis of well-defined structures. Many biological SD materials contain perfectly defined repetitions of the same unit. Being able to directly insert a larger yet monodisperse block is 
hence of great value in the synthesis of monodisperse polymers. Using the power of RAFT polymerizations, SUMI and MUMI reactions were combined, allowing for monodisperse decamers. Oligomers were in this study separated via automated flash column chromatography. This approach is significantly faster than recSEC with isolation of a 10-mer taking roughly 1h. Furthermore, the virtual separation limit of five monomer units in recSEC is greatly surpassed, now making it possible to separate higher sequence lengths up to 10 or more units easily. With no product losses encountered during purification, yields are kept at a maximum. Further utilizing the abilities of RDRP, the telechelic monodisperse decamers (10-mers) are subsequently coupled to form linear monodisperse icosamers (20-mers) by the use of RAFT end-group chemistry. This coupling not only increases the attainable chain length, it also demonstrates the plethora of click-like reactions directly available for the SUMI and MUMI products by using RDRP techniques. The herein obtained unprecedented length hence shows that RDRP techniques are highly competitive with iterative synthesis strategies for the design and synthesis of SD oligomers.

\section{Conclusions}

Overall, the use of reversible-deactivation radical polymerization techniques (or generally any controlled chain growth method) is powerful to create sequenceencoded oligomers and polymers. The dispersity of additions can be precisely tuned, ranging from polydisperse multiblock copolymers to monodisperse perfectly-structured molecules. The next aim for the research field is to find suitable applications for these materials and to make use of the unique definition of these molecules. Possible applications span wide and it will be exciting to see how the ability to control chain sequences will impact material development as a whole.

\section{Conflicts of interest}

There are no conflicts to declare.

\section{Acknowledgements}

The authors are grateful for funding from Hasselt University and Monash University.

\section{Notes and references}

1. N. G. Engelis, A. Anastasaki, G. Nurumbetov, N. P. Truong, V. Nikolaou, A. Shegiwal, M. R. Whittaker, T. P. Davis and D. M. Haddleton, Nat. Chem., 2016, 9, 171-178.

2. A. Anastasaki, V. Nikolaou, G. Nurumbetov, P. Wilson, K. Kempe, J. F. Quinn, T. P. Davis, M. R. Whittaker and D. M. Haddleton, Chem. Rev., 2016, 116, 835-877.

3. J. J. Haven, C. Guerrero-Sanchez, D. J. Keddie, G. Moad, S. H. Thang and U. S. Schubert, Polym. Chem., 2014, 5, 5236-5246.

4. J. J. Haven, C. Guerrero-Sanchez, D. J. Keddie and G. Moad, Macromol. Rapid Commun., 2014, 35, 492497.

5. G. Gody, R. Barbey, M. Danial and S. Perrier, Polym. Chem., 2015, 6, 1502-1511.

6. J.-F. Lutz, J.-M. Lehn, E. W. Meijer and K. Matyjaszewski, Nature Reviews Materials, 2016, 1, 16024.

7. J. Vandenbergh, G. Reekmans, P. Adriaensens and T. Junkers, Chem. Sci., 2015, 6, 5753-5761.

8. L. Zhang, L. R. Kucera, S. Ummadisetty, J. R. Nykaza, Y. A. Elabd, R. F. Storey, K. A. Cavicchi and R. A. Weiss, Macromolecules, 2014, 47, 4387-4396.

9. B. Wei, W. Li, Z. Zhao, A. Qin, R. Hu and B. Z. Tang, J. Am. Chem. Soc., 2017, DOI: 10.1021/jacs.6b12767.

10. W. R. Gutekunst and C. J. Hawker, J. Am. Chem. Soc., 2015, 137, 8038-8041.

11. Z. Zhang, Y.-Z. You, D.-C. Wu and C.-Y. Hong, Macromolecules, 2015, 48, 3414-3421.

12. N. G. Engelis, A. Anastasaki, G. Nurumbetov, N. P. Truong, V. Nikolaou, A. Shegiwal, M. R. Whittaker, T. P. Davis and D. M. Haddleton, Nat. Chem., 2017, 9, 171-178.

13. G. Gody, T. Maschmeyer, B. Zetterlund Per and S. Perrier, Nat. Commun., 2013, 4, 2505.

14. S. C. Solleder, R. V. Schneider, K. S. Wetzel, A. C. Boukis and M. A. R. Meier, Macromol. Rapid Commun., 2017, 38, 1600711.

15. Y. Washino and T. Michinobu, Macromol. Chem. Phys., 2014, 215, 1485-1490.

16. D. Moatsou, C. F. Hansell and R. K. O'Reilly, Chem. Sci., 2014, 5, 2246-2250.

17. Y. Ji, L. Zhang, X. Gu, W. Zhang, N. Zhou, Z. Zhang and X. Zhu, Angew. Chem. Int. Ed., 2017, 56, 2328-2333.

18. S. C. Solleder, D. Zengel, K. S. Wetzel and M. A. R. Meier, Angew. Chem. Int. Ed., 2016, 55, 1204-1207.

19. N. Zydziak, W. Konrad, F. Feist, S. Afonin, S. Weidner and C. Barner-Kowollik, Nat. Commun., 2016, 7 13672.

20. K. Takizawa, C. Tang and C. J. Hawker, J. Am. Chem. Soc., 2008, 130, 1718-1726.

21. N. ten Brummelhuis, Polym. Chem., 2015, 6, 654667.

22. M. Porel, D. N. Thornlow, C. M. Artim and C. A. Alabi, ACS Chem. Biol., 2017, 12, 715-723.

23. S. Martens, J. Van den Begin, A. Madder, F. E. Du Prez and P. Espeel, J. Am. Chem. Soc., 2016, 138, 14182 14185. 

Macromol. Rapid Commun., 2014, 35, 141-145.

25. A. Al Ouahabi, M. Kotera, L. Charles and J.-F. Lutz, ACS Macro Lett, 2015, 4, 1077-1080.

26. R. N. Zuckermann, Pept. Sci., 2011, 96, 545.

27. A. S. Knight, E. Y. Zhou, M. B. Francis and R. N. Zuckermann, Adv. Mater., 2015, 27, 5665-5691.

28. D. Mecerreyes, M. Trollsås and J. L. Hedrick, Macromolecules, 1999, 32, 8753-8759.

29. A. P. Narrainen, S. Pascual and D. M. Haddleton, J. Polym. Sci. A: Polym. Chem., 2002, 40, 439-450.

30. A. Ramakrishnan and R. Dhamodharan, Macromolecules, 2003, 36, 1039-1046.

31. D. Taton, A. Z. Wilczewska and M. Destarac, Macromol. Rapid Commun., 2001, 22, 1497-1503.

32. H. Gemici, T. M. Legge, M. Whittaker, M. J. Monteiro and S. Perrier, J. Polym. Sci A: Polym. Chem., 2007, 45, 2334-2340.

33. C. Boyer, A. H. Soeriyadi, P. B. Zetterlund and M. R. Whittaker, Macromolecules, 2011, 44, 8028-8033.

34. A. H. Soeriyadi, C. Boyer, F. Nyström, P. B. Zetterlund and M. R. Whittaker, J. Am. Chem. Soc., 2011, 133, 11128-11131.

35. A. Anastasaki, C. Waldron, P. Wilson, C. Boyer, P. B. Zetterlund, M. R. Whittaker and D. Haddleton, ACS Macro Lett., 2013, 2, 896-900.

36. C. Boyer, A. Derveaux, P. B. Zetterlund and M. R. Whittaker, Polym. Chem., 2012, 3, 117-123.

37. F. Alsubaie, A. Anastasaki, P. Wilson and D. M. Haddleton, Polym. Chem., 2015, 6, 406-417.

38. A. Simula, V. Nikolaou, A. Anastasaki, F. Alsubaie, G. Nurumbetov, P. Wilson, K. Kempe and D. M. Haddleton, Polym. Chem., 2015, 6, 2226-2233.

39. M. Garcia, M. P. Beecham, K. Kempe, D. M. Haddleton, A. Khan and A. Marsh, Eur. Polym. J., 2015, 66, 444-451.

40. A. Anastasaki, V. Nikolaou, G. S. Pappas, Q. Zhang, C. Wan, P. Wilson, T. P. Davis, M. R. Whittaker and D. M. Haddleton, Chem. Sci., 2014, 5, 3536-3542.

41. A. Anastasaki, V. Nikolaou, N. W. McCaul, A. Simula, J. Godfrey, C. Waldron, P. Wilson, K. Kempe and D. M. Haddleton, Macromolecules, 2015, 48, 14041411.

42. Y.-M. Chuang, A. Ethirajan and T. Junkers, ACS Macro Lett., 2014, 3, 732-737.

43. B. Wenn, A. C. Martens, Y. M. Chuang, J. Gruber and T. Junkers, Polym. Chem., 2016, 7, 2720-2727.

44. P. B. Zetterlund, G. Gody and S. Perrier, Macromol. Theory Simul., 2014, 23, 331-339.

45. G. Gody, T. Maschmeyer, P. B. Zetterlund and S. Perrier, Macromolecules, 2014, 47, 639-649.

46. L. Martin, G. Gody and S. Perrier, Polym. Chem., 2015, 6, 4875-4886.

47. T. G. McKenzie, Q. Fu, E. H. H. Wong, D. E. Dunstan and G. G. Qiao, Macromolecules, 2015, 48, 38643872.

48. M. Rubens, P. Latsrisaeng and T. Junkers, Polym. Chem., 2017, 8, 6496-6505.

49. G. Gody, P. B. Zetterlund, S. Perrier and S. Harrisson, Nat. Commun., 2016, 7, 10514.
50. J. Vandenbergh and T. Junkers, Macromolecules, 2014, 47, 5051-5059.

51. S. Pfeifer and J.-F. Lutz, J. Am. Chem. Soc., 2007, 129, 9542-9543.

52. D. Chan-Seng, M. Zamfir and J.-F. Lutz, Angew. Chem. Int. Ed., 2012, 51, 12254-12257.

53. M. Zamfir and J.-F. Lutz, Nat. Commun., 2012, 3, 1-8.

54. S. Srichan, H. Mutlu and J.-F. Lutz, Eur. Polym. J., 2015, 62, 338-346.

55. R. K. Roy and J.-F. Lutz, J. Am. Chem. Soc., 2014, 136, 12888-12891.

56. M. Hisano, K. Takeda, T. Takashima, Z. Jin, A. Shiibashi and A. Matsumoto, Macromolecules, 2013, 46, 7733-7744.

57. N. G. Engelis, A. Anastasaki, R. Whitfield, G. R. Jones, E. Liarou, V. Nikolaou, G. Nurumbetov and D. M. Haddleton, Macromolecules, 2018, 51, 336-342.

58. G. Nurumbetov, N. Engelis, J. Godfrey, R. Hand, A. Anastasaki, A. Simula, V. Nikolaou and D. M. Haddleton, Polym. Chem., 2017, 8, 1084-1094.

59. A. Anastasaki, B. Oschmann, J. Willenbacher, A. Melker, M. H. C. Van Son, N. P. Truong, M. W. Schulze, E. H. Discekici, A. J. McGrath, T. P. Davis, C. M. Bates and C. J. Hawker, Angew. Chem. Int. Ed., 2017, 56, 14483-14487.

60. G. Moriceau, G. Gody, M. Hartlieb, J. Winn, H. Kim, A. Mastrangelo, T. Smith and S. Perrier, Polym. Chem., 2017, 8, 4152-4161.

61. P. Radzevicius, T. Krivorotova and R. Makuska, Eur. Polym. J., 2017, 87, 69-83.

62. C. Lavilla, G. Yilmaz, V. Uzunova, R. Napier, C. R. Becer and A. Heise, Biomacromolecules, 2017, 18, 1928-1936.

63. S. n. Nishimura, N. Higashi and T. Koga, Chem - Eur. J., 2017, 23, 15050-15058.

64. J. Niu, Z. A. Page, N. D. Dolinski, A. Anastasaki, A. T. Hsueh, H. T. Soh and C. J. Hawker, ACS Macro Lett., 2017, 6, 1109-1113.

65. P. R. Judzewitsch, T. K. Nguyen, S. Shanmugam, E. H. H. Wong and C. Boyer, Angew. Chem. Int. Ed., 2018, 57, 4559-4564.

66. J. Vandenbergh, T. de Moraes Ogawa and T. Junkers, J. Polym. Sci. A: Polym. Chem., 2013, 51, 2366-2374.

67. E. Baeten, J. Haven and T. Junkers, Polym. Chem., 2017, 8, 3815-3824.

68. A. Kuroki, I. Martinez-Botella, C. H. Hornung, L. Martin, E. G. L. Williams, K. E. S. Locock, M. Hartlieb and S. Perrier, Polym. Chem., 2017, 8, 3249-3254.

69. X. Pan, S. Lathwal, S. Mack, J. Yan, S. R. Das and K. Matyjaszewski, Angew. Chem. Int. Ed., 2017, 56, 2740-2743.

70. Z. Qiao, T. Qiu, W. Liu, L. Zhang, J. Tu, L. Guo and X. Li, Polym. Chem., 2017, 8, 3013-3021.

71. K. Satoh, S. Ozawa, M. Mizutani, K. Nagai and M. Kamigaito, Nat. Commun., 2010, 1, 6.

72. Y. Hibi, S. Tokuoka, T. Terashima, M. Ouchi and M. Sawamoto, Polym. Chem., 2011, 2, 341-347.

73. Y. Hibi, M. Ouchi and M. Sawamoto, Angew. Chem. Int. Ed., 2011, 50, 7434-7437. 
74. S. Ida, T. Terashima, M. Ouchi and M. Sawamoto, J. Am. Chem. Soc., 2009, 131, 10808-10809.

75. S. Ida, M. Ouchi and M. Sawamoto, J. Am. Chem. Soc., 2010, 132, 14748-14750.

76. Y. Hibi, M. Ouchi and M. Sawamoto, Nat. Commun., 2016, 7, 11064.

77. X. Tong, B.-h. Guo and Y. Huang, Chem. Commun., 2011, 47, 1455-1457.

78. D. Oh, M. Ouchi, T. Nakanishi, H. Ono and M. Sawamoto, ACS Macro Lett., 2016, 5, 745-749.

79. P. Delduc, C. Tailhan and S. Z. Zard, Chem. Commun., 1988, 308-310.

80. B. Quiclet-Sire, G. Revol and S. Z. Zard, Tetrahedron Lett., 2010, 66, 6656-6666.

81. J. B. McLeary, F. M. Calitz, J. M. McKenzie, M. P. Tonge, R. D. Sanderson and B. Klumperman, Macromolecules, 2004, 37, 2383-2394.

82. E. T. A. v. d. Dungen, H. Matahwa, J. B. McLeary, R. D. Sanderson and B. Klumperman, J. Polym. Sci. A: Polym. Chem., 2008, 46, 2500-2509.

83. M. Chen, K. P. Ghiggino, A. W. H. Mau, E. Rizzardo, W. H. F. Sasse, S. H. Thang and G. J. Wilson, Macromolecules, 2004, 37, 5479-5481.

84. S. Houshyar, D. J. Keddie, G. Moad, R. J. Mulder, S. Saubern and J. Tsanaktsidis, Polym. Chem., 2012, 3, 1879-1889.

85. G. Moad, C. Guerrero-Sanchez, J. J. Haven, D. J. Keddie, A. Postma, E. Rizzardo and S. H. Thang, in Sequence-Controlled Polymers: Synthesis, SelfAssembly, and Properties, Am. Chem. Soc., 2014, vol. 1170, ch. 9, pp. 133-147.

An overview is given on the state-of-the-art in synthesis of sequence-controlled and sequencedefined oligomers and polymers.
86. J. J. Haven, M. Hendrikx, T. Junkers, P. J. Leenaers, A. Postma, T. Tsompanoglou, C. Boyer, J. Xu and G. Moad, ACS Symp. Ser., 2018, 1284, 77-103.

87. J. Xu, S. Shanmugam, C. Fu, K.-F. Aguey-Zinsou and C. Boyer, J. Am. Chem. Soc., 2016, 138, 3094-3106.

88. J. Xu, C. Fu, S. Shanmugam, C. J. Hawker, G. Moad and C. Boyer, Angew. Chem. Int. Ed., 2016, 129, 8496-8503.

89. C. Fu, Z. Huang, C. J. Hawker, G. Moad, J. Xu and C. Boyer, Polym. Chem., 2017, 8, 4637-4643.

90. A. Aerts, R. W. Lewis, Y. Zhou, N. Malic, G. Moad and A. Postma, Macromol. Rapid Commun., DOI: doi:10.1002/marc.201800240.

91. J. J. Haven, J. A. De Neve and T. Junkers, ACS Macro Lett., 2017, 6, 743-747.

92. J. Vandenbergh, G. Reekmans, P. Adriaensens and T. Junkers, Chem. Commun., 2013, 49, 10358-10360.

93. J. J. Haven, J. Vandenbergh and T. Junkers, Chem. Commun. , 2015, 51, 4611-4614.

94. J. J. Haven, J. Vandenbergh, R. Kurita, J. Gruber and T. Junkers, Polym. Chem., 2015, 6, 5752-5765.

95. J. J. Haven and J. Tanja, Eur. J. Org. Chem., 2017, 2017, 6474-6482.

96. J. Lawrence, S.-H. Lee, A. Abdilla, M. D. Nothling, J. M. Ren, A. S. Knight, C. Fleischmann, Y. Li, A. S. Abrams, B. V. K. J. Schmidt, M. C. Hawker, L. A. Connal, A. J. McGrath, P. G. Clark, W. R. Gutekunst and C. J. Hawker, J. Am. Chem. Soc., 2016, 138, 63066310.

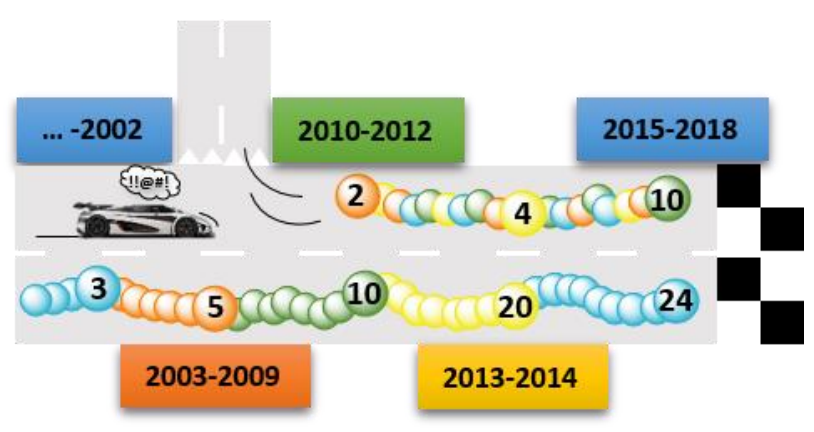

